\title{
SIMULATION AND OPTIMIZATION OF CUTTING TOOLS FOR THE POCKETS MACHINING IN 2D1/2
}

\author{
Bouaziz, Z. ; Hamza, I. ${ }^{*} \&$ Haddar, M.* \\ * Unit of Mechanics, Solids, Structures and Technological Development, \\ Ecole Supérieure des Sciences et Techniques, BP 56 Beb Mnara, 1008 Tunis, Tunisia \\ ${ }^{* *}$ Unit of Mechanics, Modeling, and Production, Ecole Nationale d'Ingénieurs de Sfax, \\ Route de Sokra Km 3.5, BP W, 3038 Sfax, Tunisia \\ E-mail: zoubeir.bouaziz@enis.rnu.tn
}

\begin{abstract}
The manufacturing time minimization is a major objective to be taken into account since the selection of machining parameters. The cutting tool diameter is one of the parameters which influence enormously the machining time of the different geometric shapes, and in particular, the pockets. In this paper, we developed an optimizing methodology of the cutting tools selection for the quadrilateral pockets machining in $2 \mathrm{D}^{1} \frac{1}{2}$. For this, we developed some analytic models expressing the path length of the cutting tool. Then, we structured an optimizing method based on the cutting time minimization. A simulation data processing tool was developed by means of MATLAB ${ }^{\circledR}$ software, which generates the cutting tool optimal diameter from the pocket geometric information. To validate our approach, we treated an application example of an ordinary quadrilateral pocket in $2 \mathrm{D} 1 / 2$.

(Received in May 2005, accepted in August 2005. This paper was with the authors 1 month for 2 revisions.)
\end{abstract}

Key Words: Machining, Pocket, Cutting Conditions, Optimization, Tool Selection

\section{Nomenclature}

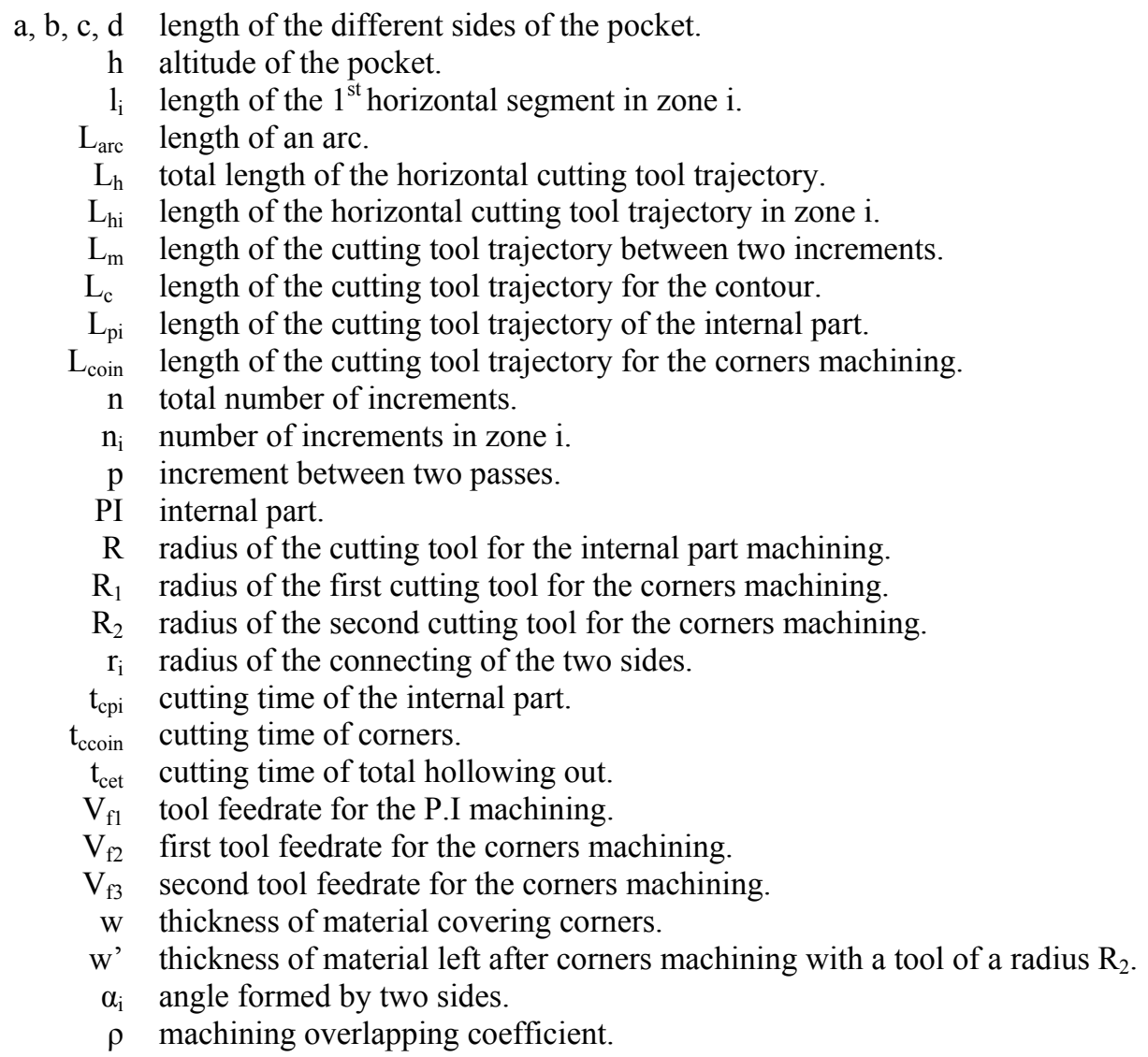




\section{INTRODUCTION}

Facing the international competition and the rapid evolution of the technological progress, enterprises must continually improve their productivity as well as the quality of their products. The computer aided design and manufacturing software have become indispensable tools, on one hand for the production automation, and on the other hand for the automatic program generating $\mathrm{CN}$. This software offers at present a wide range of operation typologies associated to generators of machining trajectories which are more performing [1]. However, a methodology allowing achieving an optimized choice of the machining process and of its different parameters becomes necessary. This will allow the CAM systems to generate the optimal trajectories of the cutting tool.

At present, several approaches have been developed [2-5] to optimize certain cutting parameters (cutting speed, feed rate, depth of pass, etc), taking into account one or several criteria. Among these approaches, we find the work of Lim and al. [6] who has described a method to determine an optimal combination of the cutting tools for the machining of a given material volume in $3 \mathrm{D}$ or of a contour in $2 \mathrm{D}$. The principle of the method consists in searching for a given contour, the accessible section for the cutting tool as well as the residual material volume left by the same tool. The everyday making use of this procedure does not give satisfactory results since several machining parameters are not taken into account in the adopted selecting process, such as the length of the tool-path, the tool engagement rate, etc.

The principle of the algorithm developed in this paper consists in modeling the cutting tool trajectory length, in order to calculate the cutting time for a given set of tools [7]. This will allows us to find the tools corresponding to the minimum values of the cutting time.

We will develop in the beginning, the analytic modeling of the cutting tool trajectory length for the machining of a quadrilateral pocket contour type 2D1/2. Afterwards we will present an optimizing method for the tools selecting. Finally, we will illustrate our approach with a numerical example by applying the modeling and the adopted optimizing method.

\section{ANALYTIC MODELING OF THE CUTTING TOOL TRAJECTORY}

\subsection{Pocket definition}

A $2 \mathrm{D}^{1} \frac{1}{2}$ quadrilateral pocket (Fig. 1) is geometrically defined by a polygonal contour having four sides. The connections of the four sides may have either similar or different radii.

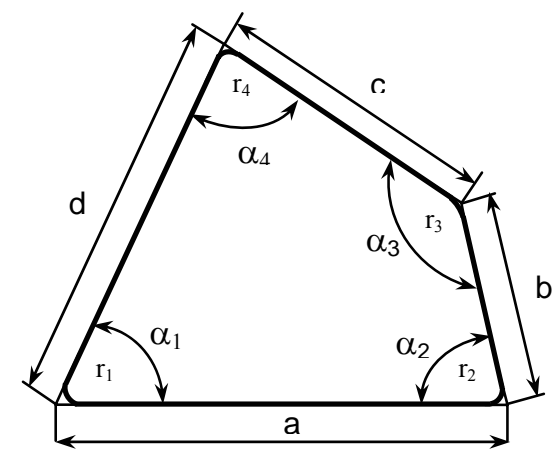

Figure 1: Parameters of a quadrilateral pocket contour.

The pocket parameters then are: four sides $\left(a, b, c\right.$ and $d$ ), four angles $\alpha_{i}$ and four connection radii $r_{i}$. Fig. 1 represents the contour of a quadrilateral pocket with the different parameters. The modelling of the preceding phase allows us to treat the pockets having a particular contour such as square, trapezium, rectangle, parallelogram and lozenge. 


\subsection{Pocket hollowing out process}

The hollowing out of a pocket is realized through two stages [8]:

- the hollowing out of a first part called internal part PI to remove the maximum of material with a tool of a radius $R-$ Fig. 2(a),

- re-starting corners correction with one or two tools - Fig. 2(b).

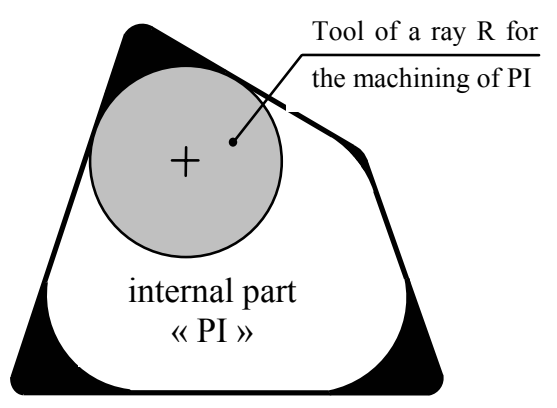

(a)

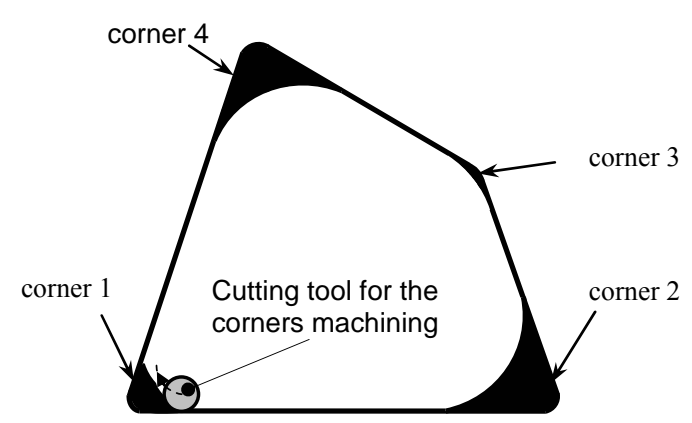

(b)

Figure 2: Principle of the pocket hollowing out.

\subsection{Machining strategy}

The hollowing out strategy adopted in our study is the Zig-Zag machining (Fig. 3). Thanks to this strategy, the sweeping is executed through passes which are parallel to a given direction leading to an alternating work in opposition and concordance. The Zig-Zag machining generates some crests of material on the pocket contour. It is necessary in this case to achieve a cycle of the pocket contour to remove this material irregularity.

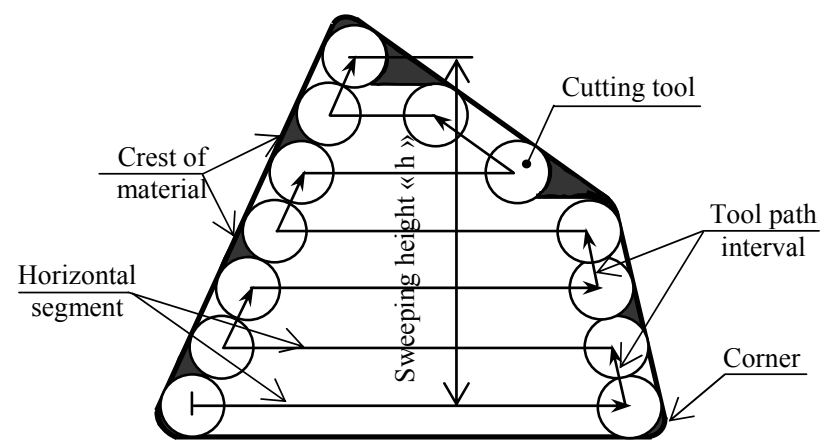

Figure 3: Zig-zag machining strategy.

\subsection{Analytic model of the cutting tool trajectory for PI}

The first stage of the machining process consists in removing the maximum of material with the first tool of a radius $R$. For this, and by applying a zig-zag machining strategy, the tool follows a trajectory made up of horizontal segments and between interval segments. The tool terminates, afterwards, with contours to remove the crests of material.

The increment defines the step of the pocket hollowing out. We search, for each tool of a radius $R$ the total altitude $h$ of the vertical sweeping up of the two zones - Fig. 4 (a). Afterwards, we determine the value of the step $p$. The altitude $h$ is expressed by the following relation:

$$
h=\left(d-R \operatorname{cotg}\left(\frac{\alpha_{1}}{2}\right)-R \operatorname{cotg}\left(\frac{\alpha_{4}}{2}\right)\right) \sin \alpha_{1}
$$



$2 \rho R$ :

The number of increments is given by the following equation by taking a step equal to

$$
n=E\left(\frac{h}{2 \rho R}\right)+1=E\left(\frac{\left(d-R \cot g\left(\frac{\alpha_{1}}{2}\right)-R \cot g\left(\frac{\alpha_{4}}{2}\right)\right) \sin \alpha_{1}}{2 \rho R}\right)+1
$$

The increment $p$ between two passes is calculated for an integer number $n$, it is expressed by the equation (3).

$$
p=\frac{\left(d-R \operatorname{cotg}\left(\frac{\alpha_{1}}{2}\right)-R \operatorname{cotg}\left(\frac{\alpha_{4}}{2}\right)\right) \sin \alpha_{1}}{n}
$$

\section{- Analytic model of horizontal segments of a total length $L_{h}$}

\section{Decomposition into zones}

To determine the trajectory length of a cutting tool of a radius $R$, we decompose the pocket internal part into two zones - Fig. 4 (a).

The length of the horizontal segments is determined by a zone at a time. We will try to find an analytic model for each zone. To calculate this length, we will take $L_{h}$ the total sum of $L_{h i}$ relating to each zone:

$$
L_{h}=L_{h 1}+L_{h 2}
$$

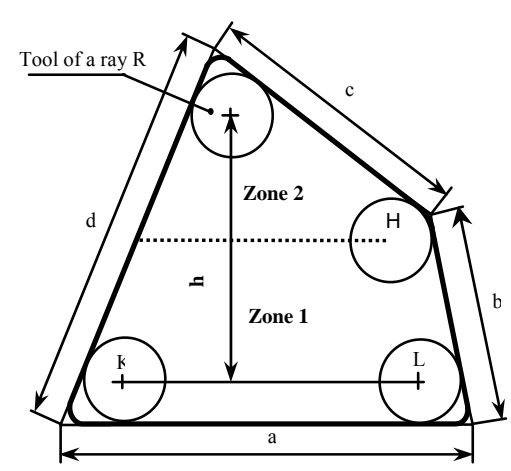

(a)

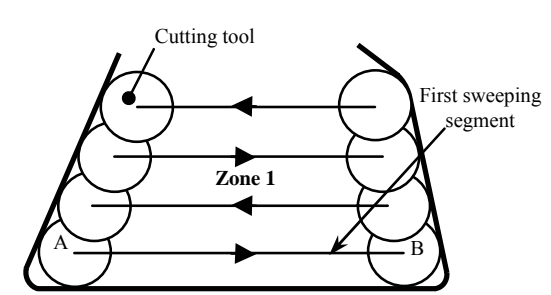

(b)

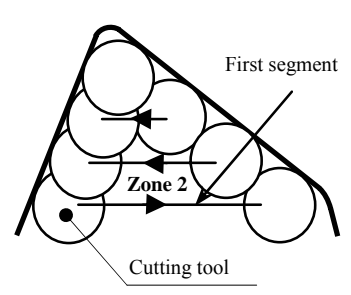

(c)

Figure 4: Decomposition into zones.

\section{Length $L_{h 1}$ of horizontal segments in zone (1)}

$\mathrm{L}_{\mathrm{h} 1}$ is the total sum of all the horizontal segments in the zone 1 - Fig. 4 (b). To modelize this length we tried to find the length of the first segment then, by instrumentation and according to the number of steps, we expressed the following model:

$$
\begin{gathered}
L_{h 1}=\sum_{i=1}^{n_{1}+1}\left[l_{1}-p \cdot(i-1) \cdot\left(\cot g \alpha_{1}+\cot g \alpha_{2}\right)\right] \\
l_{1}=a-R \cdot \cot g\left(\frac{\alpha_{1}}{2}\right)-R \cdot \cot g\left(\frac{\alpha_{2}}{2}\right)
\end{gathered}
$$

\section{Length $L_{h 2}$ of the horizontal segments in zone 2}

$L_{h 2}$ is the total sum of all the horizontal segments in the zone 2 - Fig. 4 (c). To model this length we have applied the same principle as $L_{h 1}$. The model expressing $L_{h 2}$ is the following. 


$$
L_{h 2}=\sum_{i=1}^{n 2}\left[l_{2}-p \cdot(i-1) \cdot\left(\operatorname{cotg}\left(\alpha_{1}\right)+\operatorname{cotg}\left(\pi-\left(\alpha_{1}+\alpha_{4}\right)\right)\right)\right]
$$

The length of the tool horizontal trajectory for all the internal part $L_{h}$ is expressed by the following model:

$$
L_{h}=\left[\begin{array}{l}
\sum_{i=1}^{n_{1}+1}\left[a-R \cdot \operatorname{cotg}\left(\frac{\alpha_{1}}{2}\right)-R \cdot \operatorname{cotg}\left(\frac{\alpha_{2}}{2}\right)-p \cdot(i-1) \cdot\left(\operatorname{cotg} \alpha_{1}+\operatorname{cotg} \alpha_{2}\right)\right]+ \\
\sum_{i=1}^{n 2}\left[l_{2}-p \cdot(i-1) \cdot\left(\operatorname{cotg}\left(\alpha_{1}\right)+\operatorname{cotg}\left(\pi-\left(\alpha_{1}+\alpha_{4}\right)\right)\right)\right]
\end{array}\right]
$$

\section{- Trajectory analytic model between increments}

By applying the chosen zig-zag machining strategy, the cutting tool executes a sweeping up according to passes parallel to the pocket side $a$, starting from the point $\mathrm{K}$ or from the point $\mathrm{M}$ (Fig. 5). The length $\mathrm{Lm}$ defines the trajectory length between the tool increments to move from the point $\mathrm{K}$ or $\mathrm{L}$ towards the point $\mathrm{M}$.

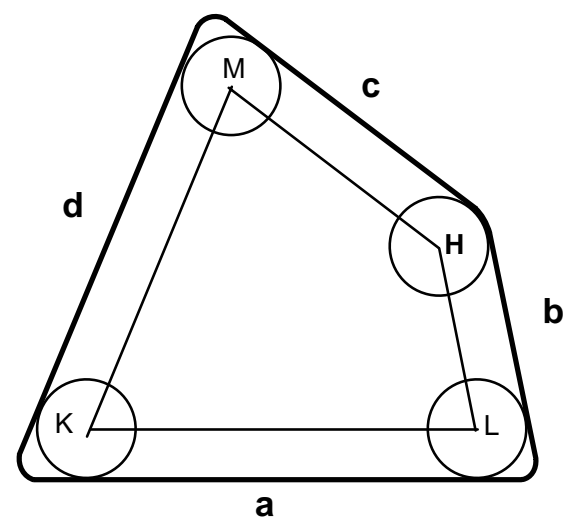

Figure 5: Between increments and contourning trajectory.

The tool trajectory length between increments is half the sum of the three segments $(\overline{\mathrm{KM}}, \overline{\mathrm{LH}}, \overline{\mathrm{HM}})$ defined in Fig. 5. We express the trajectory length between increments by the following relation:

$$
L_{m}=\frac{1}{2}\left(\frac{h}{\sin \alpha_{1}}+b-R \cdot \operatorname{cotg}\left(\frac{\alpha_{2}}{2}\right)-2 R \cdot \operatorname{tg}\left(\frac{\left(\pi-\alpha_{3}\right)}{2}\right)+c-R \cdot \operatorname{cotg}\left(\frac{\alpha_{4}}{2}\right)\right)
$$

\section{- Analytic model for the contour trajectory}

The trajectory length for the contour cycle is the total sum of the segments $(\overline{\mathrm{KL}}, \overline{\mathrm{LH}}, \overline{\mathrm{HM}}$, $\overline{\mathrm{MK}}$ ) - Fig. 5. We express the contour trajectory length by the following relation:

$$
L_{C}=\left(a-R \cdot \cot g\left(\frac{\alpha_{1}}{2}\right)-2 R \cdot \operatorname{cotg}\left(\frac{\alpha_{2}}{2}\right)+\frac{h}{\sin \alpha_{1}}+b-2 R \cdot \operatorname{tg}\left(\frac{\left(\pi-\alpha_{3}\right)}{2}\right)+c-R \cdot \operatorname{cotg}\left(\frac{\alpha_{4}}{2}\right)\right)
$$

The cutting tool total trajectory length in the internal part $L_{p i}$ is the total sum of the equations (8), (9) and (10); we will have:

$$
L_{p i}=L_{h}+L_{m}+L_{c}
$$




\subsection{Analytic model of the tool trajectory for the corners machining}

After the machining of the pocket internal part with a tool of a radius which is superior to the connections radii, it is necessary to restart the machining of the material allowance at the level of the corners with an operating resuming tool of a radius inferior or equal to the connections radii. To do this, we have developed two models for the resuming of the corners. In the first model, we use only one cutting tool for the machining of the pocket four corners, this tool must have a radius inferior or equal to the radius of the smallest connection. In the second model (Fig. 8) we propose the use of two cutting tools. A first tool (of a radius $R_{2}$ ) removes the maximum of the corner allowance. The remaining material left by the first tool will be removed by the second tool of a radius $R_{l}$.

\section{- The corners machining with only one tool}

The corners machining strategy is different from that of the internal part. The corner contour is made up of two arcs and two sides. The corner machining requires first of all the evaluation of the allowance $w$ (Fig. 6).

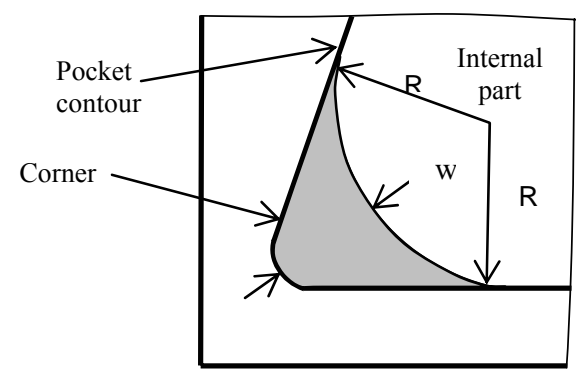

Figure 6: Definition of a corner.

The distance $w$ represents the allowance of material to remove at the level of the corners. The allowance $w$ depends on the radius $R$ of the machining cutting tool for PI, on the radius of the corner connection and also on the angle $\alpha_{i}$. The allowance of material relative to each corner « $\mathrm{i} »$ is expressed by the following relation.

$$
w_{i}=\left(R-r_{i}\right)\left(\frac{1}{\sin \left(\alpha_{i} / 2\right)}-1\right)
$$

With the purpose to find the corners resumption trajectory, our research shows that there exist several types of trajectory according to the radii of the corners resumption tool. In fact, we can distinguish the following cases of corners hollowing out:

\section{First case}

In this first case - Fig. 7 (a), the radius $R_{1}$ of the corners machining tool is equal to the connection radius and the corner thickness is inferior or equal to corners diameter multiplied by an overlapping coefficient noted $\rho(\rho<1)$. In this case we have: $r_{i}=R_{l}$ and $w_{i} \leq 2 . \rho . R_{1}$

The cutting tool trajectory length in this case is the total sum of the segments ( $\overline{\mathrm{AB}}$ et $\overline{\mathrm{BC}}$ ), it is expressed by the following relation:

$$
L_{\text {coin }}=2 \cdot\left(R-R_{1}\right) \cdot \cot g\left(\alpha_{i} / 2\right)
$$

\section{Second case}

In this second case - Fig. 7 (b), the tool radius $R_{I}$ for the corners machining is inferior to the connection radius. The thickness of the corner allowance is inferior or equal to the corners 
diameter multiplied by a coefficient of overlapping $\rho$. In this case we have: $w_{i} \leq 2 . \rho . R_{1}$ and $R_{1}<r_{i}$.

The cutting tool trajectory length is the total sum of the segments ( $\overline{\mathrm{AB}}$ and $\overline{\mathrm{CD}})$ plus the length of the arc $\mathrm{BC}=\left(\left(r_{i}-R_{l}\right)\left(\pi-\alpha_{i}\right)\right)$. The trajectory length is expressed by the following relation:

$$
L_{\text {coin }}=2 \cdot\left(R-R_{l}\right) \cdot \cot g\left(\alpha_{i} / 2\right)+\left(r_{i}-R_{l}\right)\left(\pi-\alpha_{i}\right)
$$

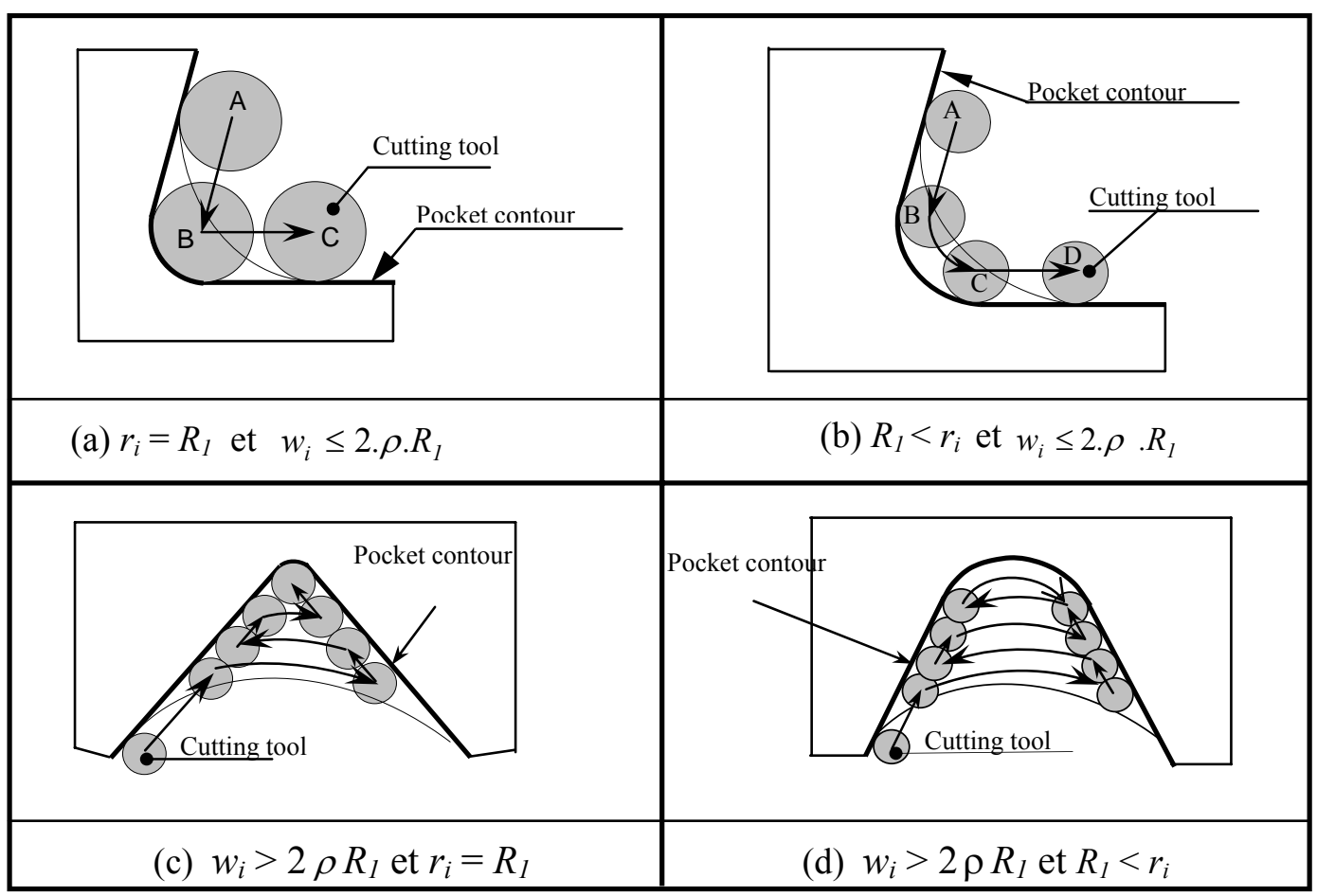

Figure 7: Cutting tool trajectory.

\section{Third case}

In this case, the tool radius $R_{l}$ is inferior or equal to the connection ray. The thickness of the corner allowance is superior to the tool diameter. In this case we have: $w_{i}>2 \rho R_{l}$ and $r_{i} \geq R_{l}$. The strategy applied in the corners machining is the zig-zag machining. The Fig. 7 (c) represents the tool trajectory in case: $R_{1}=r_{i}$. On the other hand the Fig. 7 (d) shows the cutting tool trajectory in case: $R_{l}<r_{i}$.

As for the internal part, we try to find the length of the parallel arcs, the length of the trajectory between increments and also the length of the trajectory for the border sweeping up (contourning). The number of increments or steps in a corner is expressed by the relation (15):

$$
\mathrm{n}_{\mathrm{C}}=E\left(\frac{\frac{\left(R-R_{l}\right)}{\operatorname{Sin}\left(\alpha_{i} / 2\right)}-\left(R+\rho R_{l}\right)}{2 \rho R_{l}}\right)+1
$$

The step corrected is expressed by the following relation:

$$
p_{C}=\frac{\frac{\left(R-R_{1}\right)}{\operatorname{Sin}\left(\alpha_{i} / 2\right)}-\left(R+\rho R_{1}\right)}{n_{C}}
$$


The tool path length in the third case is expressed by the following expression:

$$
L_{\text {coin }}=\left(\begin{array}{l}
\sum_{i=1}^{n_{c}}\left(\pi\left(R+\rho \cdot r_{i}(2 . i-1) \frac{\left(90-\alpha_{i} / 2-\cos ^{-1}\left(\left(R-R_{1}\right) /\left(R+\rho \cdot R_{1}(2 . i-1)\right)\right)\right)}{90}\right)\right)+ \\
3\left(R-R_{1}\right) \operatorname{cotg}\left(\alpha_{i} / 2\right)+\left(r_{i}-R_{1}\right)\left(\frac{\left(\pi-\alpha_{i}\right)}{2}\right)
\end{array}\right)
$$

\section{- The machining of the corners with two tools}

The corner cutting time increases when the thickness of the corner allowance becomes important (the tool radius of PI $>>$ to the connection radius) - Fig. 8. To remedy to this problem, we propose an approach which consists in machining:

1 - with a tool of a radius superior to the difference of allowances,

2- with a second tool of a radius inferior or equal to the smallest connection radius.

The second tool diameter must slightly exceeds the difference of allowances $\left(2 R_{2}>w-w^{\prime}\right)$. The allowance $w^{\prime}$ is expressed by the following relation:

$$
w_{i}^{\prime}=\left(R_{2}-r_{i}\right)\left(\frac{1}{\sin \left(\alpha_{i} / 2\right)}-1\right)
$$

The tool trajectory length (2) is expressed by the following relation:

$$
L_{\text {coin2 } 2}\left(\operatorname{tool}_{2}\right)=2\left(R-R_{2}\right) \cdot \operatorname{cotg}\left(\alpha_{i} / 2\right)
$$

The trajectory length of the cutting tool for the corner hollowing out is expressed by the following relation:

$$
L_{\text {coin }}=L_{\text {coin } 1}+L_{\text {coin } 2}
$$

where, $L_{\text {coinl } 1}$ is expressed by the model (17).

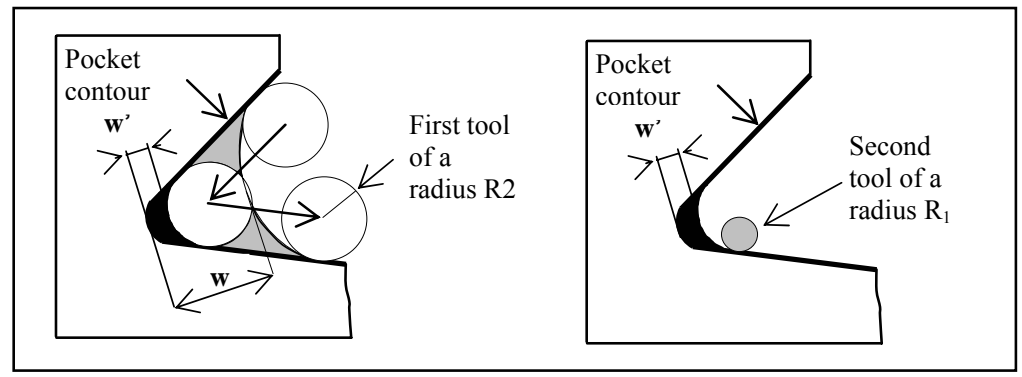

Figure 8: Corners machining with two tools.

\subsection{Objective functions}

The method of the optimal determination of the cutting parameters is based on the objective functions, which consists in finding the production minimum cost or time. In this study, we have formulated the machining time models for each cycle. They are used as objective functions. These mathematical models are expressed by the relation below:

- The machining of corners with only one tool, the total machining time is equal to :

$$
t_{c e t}=\frac{L_{p i}}{V_{f 1}}+\frac{L_{c o i n}}{V_{f 2}}
$$

- The machining of corners with two tools, the total time is equal to :

$$
t_{c e t}=\frac{L_{p i}}{V_{f 1}}+\frac{L_{c c o i n} 1}{V_{f 2}}+\frac{L_{c c o i n} 2}{V_{f 3}}
$$




\section{THE OPTIMIZING METHODOLOGY}

The methodology of selecting the cutting tools in our study is decomposed into two essential stages. In the first stage we choose the diameter of the cutting tool for the corners hollowing out, and this is according to radii of the pocket connection as well as the cutting tools. In the second stage, we will find the diameter of the cutting tool relating to the minimum time for the internal part machining. The optimizing methodology is described by the flow chart (Fig. 9).

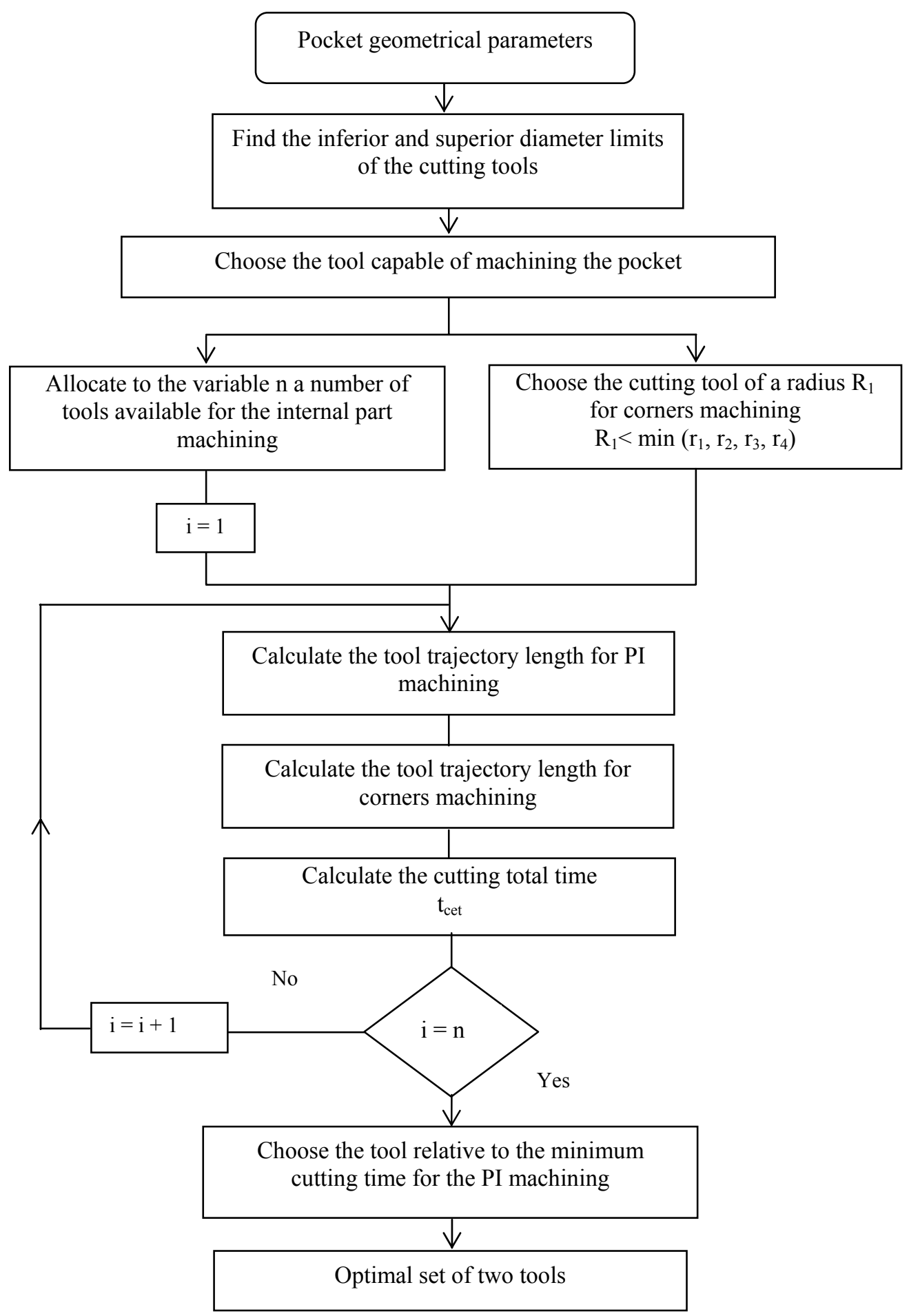

Figure 9: Optimizing flowchart of the tool selection (pocket machining with two tools). 


\section{TREATMENT OF A POCKET EXAMPLE}

\subsection{Pocket geometrical parameters}

We propose to study a piece which consists of a quadrilateral pocket of an ordinary contour and identical connection radii. The geometrical parameters defining the validation pocket of Fig. 1 are presented in Table I. The material of the piece: non-alloyed steel with a resistance $500<R_{m}<800 \mathrm{~N} / \mathrm{mm}^{2}$.

Table I: Pocket parameters.

\begin{tabular}{|c|c|c|c|c|c|c|c|c|c|c|c|}
\hline \multicolumn{4}{|c|}{ Sides } & \multicolumn{4}{c|}{ Angles } & \multicolumn{3}{c|}{ Radius of connection } \\
\hline $\begin{array}{c}a \\
(\mathrm{~mm})\end{array}$ & $\begin{array}{c}b \\
(\mathrm{~mm})\end{array}$ & $\begin{array}{c}c \\
(\mathrm{~mm})\end{array}$ & $\begin{array}{c}d \\
(\mathrm{~mm})\end{array}$ & $\alpha_{1}{ }^{\circ}$ & $\alpha_{2}{ }^{\circ}$ & $\alpha_{3}{ }^{\circ}$ & $\alpha_{4}{ }^{\circ}$ & $\begin{array}{c}r_{1} \\
(\mathrm{~mm})\end{array}$ & $\begin{array}{c}r_{2} \\
(\mathrm{~mm})\end{array}$ & $\begin{array}{c}r_{3} \\
(\mathrm{~mm})\end{array}$ & $\begin{array}{c}r_{4} \\
(\mathrm{~mm})\end{array}$ \\
\hline 80 & 38 & 54 & 68 & 72.9 & 66.8 & 146.9 & 73.4 & 4 & 4 & 4 & 4 \\
\hline
\end{tabular}

\subsection{Cutting conditions}

We consider in this example a set of tools consisting of 14 tools. We'll present in Table II, the different tools parameters as well as the associated numbering. The tools are numbered from 1 to 16 following the increasing order of diameters.

Table II is extracted from the book Memotech [9], where $d$ represents the diameter of the tool in $\mathrm{mm}, z$ the number of the tool teeth, $f_{z}$ feed rate per tooth in $\mathrm{mm} / \mathrm{rev}$. These values are given for the machining of non-alloyed steels, of general usage for thermal treatment and for tool life of $60 \mathrm{~min}$, the cutting speed $V_{c}=28 \mathrm{~m} / \mathrm{min}$. The tool is of steel (ARS).

Table II: Cutting tool and conditions.

\begin{tabular}{|c|c|c|c|c|c|c|c|c|c|c|c|c|c|c|}
\hline $\mathbf{N}^{\circ}$ tool & 1 & 2 & 3 & 4 & 5 & 6 & 7 & 8 & 9 & 10 & 11 & 12 & 13 & 14 \\
\hline $\mathbf{d ~ ( m m )}$ & 8 & 10 & 12 & 14 & 16 & 18 & 20 & 22 & 25 & 28 & 30 & 32 & 36 & 40 \\
\hline $\mathbf{z}$ & 4 & 4 & 4 & 4 & 4 & 4 & 4 & 4 & 4 & 5 & 5 & 5 & 6 & 6 \\
\hline $\mathbf{f}_{\mathbf{z}}\left(\mathbf{1 0} \mathbf{1 0}^{-3} \mathbf{m m} / \mathbf{r e v}\right)$ & 13 & 20 & 25 & 30 & 35 & 40 & 45 & 50 & 55 & 50 & 50 & 55 & 55 & 55 \\
\hline
\end{tabular}

\subsection{Manufacturing constraints}

The cutting tool minimum radius must be at least equal to the largest connection ray, in other words: $R_{\min }=4 \mathrm{~mm}\left(d_{\min i}=8 \mathrm{~mm}\right)$. The choice of the cutting tool maximum radius must verify the following condition: The cutting tool must come within the scope of the pocket contour. In this example $d_{\max }=50 \mathrm{~mm}$.

The radius of the cutting tool for the corners machining must be inferior or equal to the smallest connection radius. For this example, we consider $R_{l}=4 \mathrm{~mm}$ (last tool) since all the connections have a radius equal to $4 \mathrm{~mm}$.

\subsection{Optimizing of the cutting tool selection: numerical results}

For our problem solution, we developed a MATLAB ${ }^{\circledR}$ software data-processing application. The application of this program allowed us to get machining time results for the different cutting tools.

\section{- The machining of a pocket with only one tool}

We will treat in this case the machining of a pocket with only one cutting tool to demonstrate the effect of the number of tools. In this example, we considered a tool radius equal to the 
radii of the connections. After generating numerical values we get a pocket cutting time equal to $10.29 \mathrm{~min}$.

\section{- The machining of a pocket with two tools}

After choosing the first under roughing tool for the corners machining, we will try to find a second tool for the pocket machining. This tool will be used for the internal part machining. The variation of the total cutting time according to the radius $R$, is represented by the Fig. 10 (a).

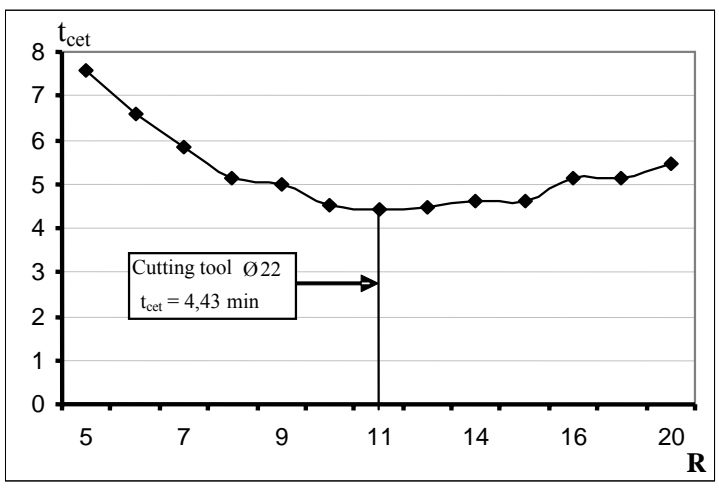

(a)

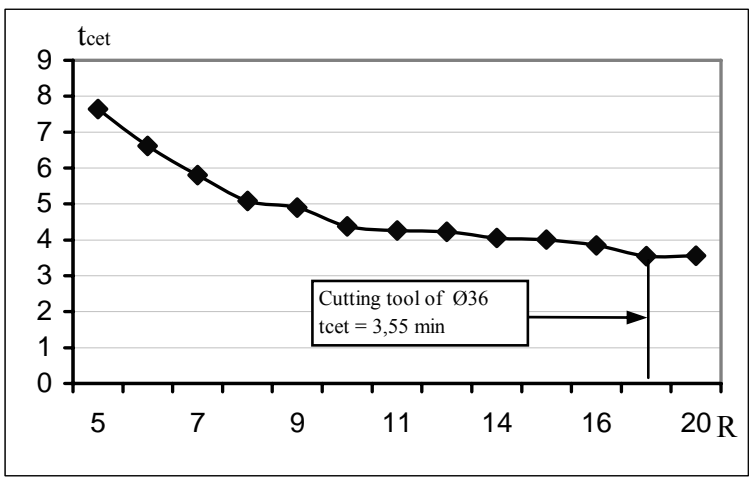

(b)

Figure 10: Variation of the total cutting time $t_{c e t}$ according to $R$.

The minimum cutting time $(4.43 \mathrm{~min})$ is obtained by the tools $\left(\mathrm{n}^{\circ} 8\right.$ of $\varnothing 22 \mathrm{~mm}$ and $\mathrm{n}^{\circ} 1$ de $\varnothing 8 \mathrm{~mm}$ ), we also notice that we can use other couples of tools which allow a cutting time very near the optimal time such as: $\left(\mathrm{n}^{\circ} 9\right.$ of $\varnothing 25 \mathrm{~mm}, \mathrm{n}^{\circ} 1$ of $\left.\varnothing 8 \mathrm{~mm}\right),\left(\mathrm{n}^{\circ} 7\right.$ of $\varnothing 20 \mathrm{~mm}, \mathrm{n}^{\circ} 1$ of $\varnothing 8 \mathrm{~mm}),\left(\mathrm{n}^{\circ} 10\right.$ of $\varnothing 28 \mathrm{~mm}, \mathrm{n}^{\circ} 1$ of $\left.\varnothing 8 \mathrm{~mm}\right),\left(\mathrm{n}^{\circ} 11\right.$ of $\varnothing 30 \mathrm{~mm}, \mathrm{n}^{\circ} 1$ of $\left.\varnothing 8 \mathrm{~mm}\right)$.

\section{- The machining of a pocket with three tools}

In our paper, we have developed a method for the use of three cutting tools. The principle consists in machining the internal part with a tool and the corners with two tools (the second tool diameter must slightly exceed the differences of the corners thicknesses $2 R_{2}>\left(w-w^{\prime}\right)$ ), we try to find for the largest tool available, the difference of allowances (w-w'), afterwards, we will choose the nearest and slightly superior diameter. In our case, we have executed our operations with a tool of a radius $R_{2}=6 \mathrm{~mm}$.

The corners were machined with the tools $n^{\circ} 1$ and $n^{\circ} 3$. The variation of the total cutting time according to the tool radius $R$ is represented by Fig. 10 (b).

\section{CONCLUSION}

In our approach, we adopted the decomposition of the machining process into two stages. A first stage consists in removing the maximum volume of material with a tool of a radius superior to the radii of the connections. On the other hand, the second stage allows us to make connections resumption with a tool of a radius inferior or equal the radii of connections.

We treated an example to illustrate the approach for the selection of an optimal set of tools. In fact, we emphasized the effect of using several cutting tools upon the machining time.

The obtained results are very satisfactory (rapid generating of optimal values for the cutting tools diameters, results depending on the constraints and on the criteria previously 
determined, etc.). These results can be refined and improved by integrating the rapid advances and the time of the cutting tools changing in the modelling.

\section{REFERENCES}

[1] Pateloup, V.; Duc, E.; Ray, P. (2004). Corner optimization for pocket machining, International Journal of Machine Tools and Manufacture, Vol. 44, 1343-1353

[2] Wang, J.; Kuriyagawa, T.; Wei, X. P.; Guo, D. M. (2002). Optimization of cutting conditions for single pass turning operations using a deterministic approach, International Journal of Machine Tools and Manufacture, Vol. 42, 1023-1033

[3] Yan, X.; Shirase, K.; Hirao, M.; Yasui, T. (2000). Extraction and optimisation of cutting conditions for $2^{1 / 2}$ axis milling, International Journal of Advanced Manufacturing technology, Vol. 16, 695-699

[4] Amiolemhen, P. E.; Ibhadode, A. O. A. (2004). Application of genetic algorithms - determination of the optimal machining parameters in the conversion of a cylindrical bar stock into a continuous finished profile, International Journal of Machine Tools and Manufacture, Vol. 44, 1403-1412

[5] Cus, F. (2000). The inclusion of the geometrical shape of the cutter into the optimisation of the milling process, International Journal of Advanced Manufacturing technology, Vol. 16, 392-403

[6] Lim, T.; Corney, J.; Ritchie, J. M.; Clark, D. E. R. (2000). Optimising automatic tool selection for $2^{1 / 2} \mathrm{D}$ components, ASME 2000 Design Engineering Technical Conference and Computers and Information in Engineering Conference Proceedings, Baltimore, Maryland

[7] Dereli, T.; Filiz, I. H.; Baykasoglu, A. (2001). Optimizing cutting parameters in process planning of prismatic parts by using genetic algorithms, International Journal of Production Research, Vol. 39, No. 15, 3303-3328

[8] Bouaziz, Z.; Ben Younes, J.; Zghal, A. (2004). Methodology of machining costs evaluation for die and mold manufacturing, Journal of Materials Processing Technology, Vol. 152, Issue 2, 237-245

[9] Barlier, C. ; Poulet, B. (1999). Mémotech génie mécanique - productique mécanique, Deuxième édition - Editions CASTEILLA 